\title{
Upregulated LAMB3 increases proliferation and metastasis in thyroid cancer
}

This article was published in the following Dove Press journal:

OncoTargets and Therapy

\author{
Yinghao Wang* \\ Yixiang Jin* \\ Adheesh Bhandari \\ Zhihan Yao \\ Fan Yang \\ Yiyuan Pan \\ Zhouci Zheng \\ Shixu Lv \\ Ouchen Wang
}

Department of Surgical Oncology, The First Affiliated Hospital of

Wenzhou Medical University,

Wenzhou, Zhejiang, People's

Republic of China

*These authors contributed equally to this work
Correspondence: Ouchen Wang; Shixu Lv

Department of Surgical Oncology, The First Affiliated Hospital of Wenzhou Medical University, Nanbai Xiang, Ouhai, Wenzhou 325000, Zhejiang, People's

Republic of China

Tel +86 I39 57706099 ;

+86 I58 25420367

Emailwoconcology2000@।63.com; Ivshixu@outlook.com
Background: Thyroid cancer is the most commonly reported endocrine malignancy, and its increased incidence has been the highest in all human tumors in recent decades. To investigate the mechanism of papillary thyroid cancer (PTC) occurrence and progression, we performed RNA sequencing and found an upregulated gene, $L A M B 3$. However, the biological function of $L A M B 3$ is still not clear.

Materials and methods: We analyzed $L A M B 3$ expression using The Cancer Genome Atlas (TCGA) database and hypothesized $L A M B 3$ to be a gene associated with PTC. To test this hypothesis, we collected 89 pairs of thyroid nodules and adjacent normal thyroid tissues (56 pairs of PTCs, 33 pairs of benign thyroid nodules). Afterward, we performed real-time quantitative polymerase chain reaction (RT-qPCR) to investigate $L A M B 3$ expression in thyroid nodule patients, and then analyzed clinicopathologic features. We performed proliferation, colony formation, migration, and invasion assays to determine the function of $L A M B 3$ in PTC.

Results: We demonstrated that $L A M B 3$ plays oncogenic roles in PTC. The relative expression of $L A M B 3$ is significantly upregulated in PTC compared with matched thyroid normal tissues in validated cohort and TCGA cohort $(P<0.001)$. We also checked area under the curve (AUC of receiver operator characteristic [ROC]) of $97.3 \%$ for validated cohort and $90.1 \%$ for TCGA cohort to differentiate PTC tumors from normal tissues. In clinicopathologic feature analysis, we found that upregulated $L A M B 3$ is closely related to lymph node metastasis $(P=0.018)$. Furthermore, knockdown of $L A M B 3$ inhibited the proliferation, colony formation, migration, and invasive capacity of PTC.

Conclusion: This study indicated that $L A M B 3$ is a gene associated with PTC.

Keywords: papillary thyroid cancer, $L A M B 3$, PTC

\section{Introduction}

Thyroid cancer is the most commonly reported endocrine malignancy, and its increased incidence has been the highest in all human tumors in recent decades. ${ }^{1,2}$

Thyroid cancer incidence has increased at a rate of $4 \%$ annually and exhibited a triple increase over the past decades in many countries. ${ }^{1,3}$ Due to the widespread use of neck ultrasonography, some histological types of thyroid cancer can be diagnosed during the early stage and prevented from developing to severe clinical disease. However, large and advanced thyroid cancer keeps increasing in incidence. ${ }^{4-6}$

Thyroid cancer is classified into five histological types, namely parafollicular C-cell-derived medullary thyroid cancer, follicular cell-derived anaplastic thyroid cancer, poorly differentiated thyroid cancer, follicular thyroid cancer, and papillary thyroid cancer (PTC); these types classically account for 3\%, 2\%, 5\%, 10\%, and $~ 80 \%$ of all thyroid malignancies, respectively. ${ }^{7}$ Furthermore, the genetic alterations are significantly associated with the tumorigenesis of thyroid cancer and prognosis of thyroid 
cancer patients. Genetic rearrangements, including the RET proto-oncogene, are certainly common in PTC (3\%-60\%). ${ }^{8,9}$ The rearrangement gene, such as RET/PTC1 and RET/PTC3, can continuously activate the RET tyrosine kinase domain, which is important in triggering changes in the cytoplasm of PTC cells. ${ }^{10,11}$ Furthermore, genetic mutations can drive tumorigenesis and progression of thyroid cancer, including BRAF mutation, ${ }^{12,13}$ RAS mutation, ${ }^{14,15}$ TERT mutation, ${ }^{16,17}$ and P53 mutation. ${ }^{18}$

We have made progress in early diagnosis and surgical treatment and found some driver mutations in tumorigenesis and development; however, the molecular mechanism of PTC is still undetermined. To explore the occurrence and progression of thyroid cancer, we performed RNA sequencing (RNA-seq) of 19 pairs of primary PTC tissues and adjacent normal tissues. After analyzing the data, the $L A M B 3$ gene appeared to be a gene associated with PTC that is crucial in tumorigenesis.

Tumor invasion and metastasis are significantly associated with the extracellular matrix (ECM) and the tumor's process of breaking down the basement membrane (BM) and contribute to the poor prognosis of thyroid cancer patients. Laminin (LM), one of the most common basement membrane, is made up of three different chains $(\alpha, \beta$, and $\gamma)$ that are encoded in human beings by $5 \alpha, 4 \beta$, and $3 \gamma$ genes. ${ }^{19}$ In mammals, researchers recently identified 15 different LM isoforms. ${ }^{20} L M-332 / L M-5$ is encoded by $L A M A 3, L A M B 3$, and $L A M C 2$, which consists of $\alpha 3, \beta 3$, and $\gamma 2$. LM-332 composes the ECM that affects cell adhesion, proliferation, migration, and differentiation. ${ }^{21}$ However, the function of the $L A M B 3$ gene is still unknown in PTC. Some researchers have demonstrated a significant association between $L A M B 3$ and clinical characterization; ${ }^{22,23}$ however, its importance in PTC remains to be investigated. In this study, LAMB3 was significantly upregulated in PTC compared with adjacent normal tissue upon RNA-seq and bioinformatics analysis. In addition, $L A M B 3$ is a gene associated with PTC and a potential diagnostic marker in PTC.

\section{Patients and methods}

\section{Patients and samples}

A total of 56 pairs of matched PTC and adjacent normal tissues and 33 pairs of matched benign thyroid nodules and adjacent normal tissues were collected during initial surgery. Samples after surgical resection were immediately snap frozen in liquid nitrogen and stored at $-80^{\circ} \mathrm{C}$ before extraction. All samples were confirmed by postoperative histopathological examination.
Histopathological types of all cases were confirmed by two pathologists. All procedures performed in our study involving human participants were approved by the ethics committee of the First Affiliated Hospital of Wenzhou Medical University. Written informed consent for the scientific use of the biological material was obtained from each patient. The $L A M B 3$ expression values (transcripts per million, TPM) of thyroid cancer were downloaded from The Cancer Genome Atlas (TCGA) database (TCGA-thyroid carcinoma project, 59 pairs of thyroid cancer with adjacent normal thyroid tissues for expression level; 375 thyroid cancers with complete clinicopathologic features for further analysis, including age, gender, tumor size, lymph node metastasis, clinical stage, and histological type).

\section{Next-generation sequencing and bioinformatics analysis}

Total RNA was extracted from tissue samples using Trizol reagent (Thermo Fisher Scientific, Waltham, MA, USA) according to the manufacturer's protocol. After the quality test, cDNA libraries were prepared using Ion Total RNA-Seq Kit v2.0 (Thermo Fisher Scientific) according to the manufacturer's instructions. They were then processed for sequencing using Illumina HiSeq 2500 according to commercially available protocols. We applied EBSeq algorithm to screen out the differently expressed genes using the following criteria: 1) fold change $>2$ for upregulation or downregulation, 2) false discovery rate $<0.05$, and 3) $P$-value $<0.05$.

\section{Cell lines and cell culture}

The TPC1 and BCPAP cell lines were provided by Professor Mingzhao Xing of the Johns Hopkins University School of Medicine, Baltimore, MA, USA. The KTC-1 cell line was provided by Stem Cell Bank, Chinese Academy of Sciences (Beijing, People's Republic of China). The TPC1 and BCPAP were grown in Roswell Park Memorial Institute 1640 medium (RPMI1640; Thermo Fisher Scientific; 10\% [v/v] fetal bovine serum [FBS], $1 \times$ sodium pyruvate, $1 \times$ minimum essential medium nonessential amino acids, $37^{\circ} \mathrm{C}, 5 \% \mathrm{CO}_{2}$ ). KTC-1 was grown in RMPI1640 (10\% [v/v] FBS, $1 \times$ minimum essential medium nonessential amino acids, $37^{\circ} \mathrm{C}, 5 \% \mathrm{CO}_{2}$ ).

These cells used in this study obtained ethical approval from the institutional review board of the First Affiliated Hospital of Wenzhou Medical University (approval no 2012-57).

\section{RNA isolation and real-time quantitative polymerase chain reaction}

RNA isolation was performed using Trizol reagent according to the manufacturer's manual. Reverse transcription was 
performed with ReverTra Ace qPCR RT Kit (Toyobo, Osaka, Japan), using oligo-dT-primers and $1 \mu \mathrm{g}$ mRNA per reaction as a template. Real-time quantitative polymerase chain reaction (RT-qPCR) analysis was performed in triplicate on the ABI Prism 7500 Sequence Detection System (Thermo Fisher Scientific) using the Thunderbird SYBR qPCR Mix (Toyobo) according to the manufacturer's recommendations. The relative expression of $L A M B 3$ was normalized by GADPH expression levels. Primer sequences were as follows: LAMB3: 5'-GCAGCCTCACAACTACTACAG-3' (forward) and 5'-CCAGGTCTTACCGAAGTCTGA-3' (reverse); GAPDH: 5'-GGTCGGAGTCAACGGATTTG-3' (forward) and 5'-ATGAGCCCCAGCCTTCTCCAT-3' (reverse).

\section{RNA interference}

Small interfering RNA (siRNA) for $L A M B 3$ and nontargeting siRNA (negative control [NC] siRNA) were purchased from GenePharma (Shanghai, People's Republic of China) for siRNA-mediated gene knockdown. Cell lines were seeded in 12-well plates and were incubated overnight. Cells that were $30 \%$ confluent were transfected by RNAiMAX (Thermo Fisher Scientific) with a final siRNA concentration of $100 \mathrm{nM}$ according to the manufacturer's protocol. Cells were harvested $48 \mathrm{~h}$ after transfection of RNA isolation. All assays were performed in triplicate. The siRNA sequences were as follows: siRNA1: 5'-CCAAAGGUGCGACUGCAAUTT-3' (forward) and 5'-AUUGCAGUCGCACCUUUGGTT-3' (reverse); siRNA2: 5'-CCAGCGAGGCUACUGUAAUTT-3' (forward) and 5'-AUUACAGUAGCCUCGCUGGTT-3' (reverse).

\section{Cell proliferation assay}

TPC1 $\left(2 \times 10^{3}\right.$ cells $)$, KTC- 1 , and BCPAP cells $\left(3 \times 10^{3}\right.$ cells $)$ were seeded in 96-well plates overnight. On the next day, all cells were transfected with siRNA. Cell Counting Kit-8 (CCK-8; Keygen, Nanjing, People's Republic of China) was added to each well. After 2-h incubation, the plate was shaken and measured using SpectraMax M5 (Molecular Devices LLC, Sunnyvale, CA, USA) at $450 \mathrm{~nm}\left(\mathrm{OD}_{450}\right)$. This same procedure was repeated every $24 \mathrm{~h}$ until the last plate was measured. All assays were repeated in triplicate.

\section{Colony formation assay}

The three transfected cells or control cells $\left(2 \times 10^{3}\right.$ cells for TPC1, $3 \times 10^{3}$ cells for BCPAP and KTC-1) were seeded in six-well plates and incubated for 8-12 days. Subsequently, each well was washed thrice with PBS, fixed with 4\% paraformaldehyde (Sigma-Aldrich Co., St Louis, MO, USA) for
$30 \mathrm{~min}$, and stained with $0.01 \%$ crystal violet for $30 \mathrm{~min}$. All assays were repeated in triplicate.

\section{Migration and invasion assays \\ Cell migration assay}

Cells $\left(2 \times 10^{4}\right.$ for TPC1, $3 \times 10^{4}$ for BCPAP and KTC-1) were suspended in $200 \mu \mathrm{L}$ serum-free RPMI1640 and seeded into the upper chamber (Corning Incorporated, Corning, NY, USA) of each insert. Subsequently, $500 \mu \mathrm{L}$ of RPMI1640 containing $10 \%$ FBS was added to a 24 -well plate. After incubation at $37^{\circ} \mathrm{C}$ for $24 \mathrm{~h}$, the cells that migrated were fixed and stained for 30 min with $0.01 \%$ crystal violet solution in PBS.

\section{Cell invasion assay}

Chambers were uniformly covered with $60 \mu \mathrm{L}$ Matrigel diluted with RPMI1640 to a certain percentage and incubated at $37^{\circ} \mathrm{C}$ for $4 \mathrm{~h}$. Subsequently, cells $\left(4 \times 10^{4}\right.$ for TPC1, $6 \times 10^{4}$ for BCPAP and KTC-1) were suspended in $200 \mu \mathrm{L}$ RPMI1640 and seeded in the upper chambers, and $500 \mu \mathrm{L}$ RPMI1640 containing 10\% FBS was added to the lower chamber. After incubation at $37^{\circ} \mathrm{C}$ for $24 \mathrm{~h}$, the cells were fixed and stained.

\section{Statistical analysis}

Data on normal distribution are expressed as mean \pm SD. Categorical variables were expressed as the percentage and were compared with chi-square test or Fisher's exact test, as appropriate. Otherwise, differences between groups were analyzed using Student's $t$-test (two-tailed). The ability of single gene expression to discriminate (concordance) was determined by calculating the area under the curve (AUC) of the receiver operator characteristic (ROC) curve. All $P$-values were two sided, and $P$-value $<0.05$ was considered statistically significant. Statistical analysis was performed using SPSS software version 23.0 (IBM Corporation, Armonk, NY, USA). GraphPad Prism7 (GraphPad Software, Inc., La Jolla, CA, USA) was used for constructing the graphs.

\section{Results \\ LAMB3 expression was upregulated in PTC}

In our previous study, we performed RNA-seq of 19 pairs of primary PTC tissues and adjacent normal tissues. By further analysis, we found that $L A M B 3$ was significantly upregulated in our data (Figure 1A), which is according to the expression in TCGA database (59 pairs of thyroid cancer with adjacent normal thyroid tissues; Figure 1B). Subsequently, we detected 

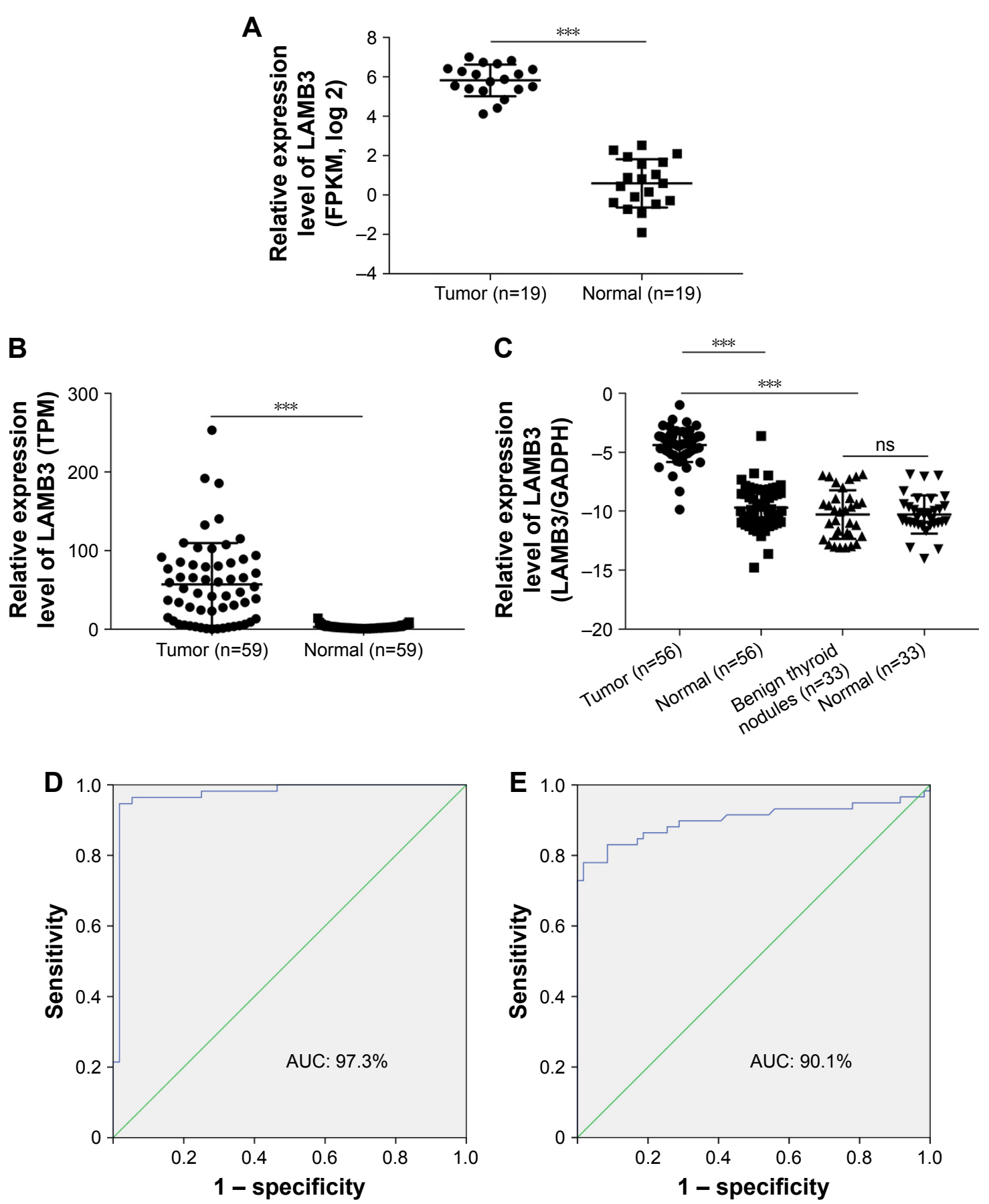

Figure I LAMB3 relative expression was significantly upregulated in thyroid cancer samples compared with normal thyroid tissues in validated cohort and TCGA cohort. Notes: (A) LAMB3 expression was upregulated by 19 pairs of matched thyroid cancer and normal tissues by RNA-seq (***P $<0.01$ ). (B) LAMB3 relative expression was validated by 59 pairs of matched thyroid cancer and normal tissues by TGCA database $(* * * P<0.01)$. (C) LAMB3 expression was examined in 56 paired PTC samples with adjacent normal tissues and 33 benign thyroid nodules with adjacent normal tissues by RT-qPCR (***P<0.0 I). (D) LAMB3 expression level for differentiating normal tissue from PTC in our validated cohort (59 pairs of PTC tissues with adjacent normal tissues); AUC: $97.3 \%$, sensitivity: $94.6 \%$, and specificity: $98.2 \%$ in the validated cohort. (E) LAMB3 expression level for differentiating normal tissue from PTC in TCGA cohort (507 PTC tissues and 59 normal tissues), AUC: $90.1 \%$, sensitivity: 83.1\%, and specificity: $91.5 \%$ in TCGA cohort.

Abbreviations: AUC, area under the curve; ns, no sense; PTC, papillary thyroid cancer; RNA-seq, RNA sequencing; RT-qPCR, real-time quantitative polymerase chain reaction; TPM, transcripts per million; TCGA, The Cancer Genome Atlas.

the relative expression of $L A M B 3$ in 89 pairs of matched thyroid nodule tissues (56 pairs of PTCs and 33 pairs of benign thyroid nodules) with normal tissues by RT-qPCR as the validated cohort. As shown in Figure 1C, the relative expression of $L A M B 3$ in tumor tissues was significantly upregulated compared with the matched normal tissues $(P<0.001)$ and benign thyroid nodules $(P<0.001)$. However, there is no significant difference compared with the expression of benign 
thyroid nodules and adjacent normal thyroid tissues $(P>0.1)$. Then, we performed an ROC curve to evaluate the diagnostic potential of $L A M B 3$ expression level. In our validated cohort (56 pairs of PTC tissues with normal tissues), ROC curve analysis indicated that $L A M B 3$ expression level can be a promising marker for differentiating malignant thyroid cancer from normal tissue, with a sensitivity and specificity of $94.6 \%$ and $98.2 \%$, respectively (an area under the ROC curve: $97.3 \%$; Figure 1D). This result is further confirmed by TCGA data (507 PTC tissues and 59 normal tissues) set with a sensitivity and specificity of $83.1 \%$ and $91.5 \%$, respectively (an area under the ROC curve: 90.1\%; Figure 1E). These results demonstrated that $L A M B 3$ is upregulated and associated with PTC.

\section{LAMB3 expression is associated with clinicopathologic features}

To investigate whether $L A M B 3$ is related to PTC "occurrence" and progression, we analyzed the clinical features with the relative expression of $L A M B 3$. Based on the 56 pairs of PTC tissues with matched normal tissues, patients were classified into a low- and a high-expression group according to the median value. In TCGA cohort, results revealed that lymph node metastasis $(P<0.001)$ and histological type $(P<0.001)$ were significantly related to high $L A M B 3$ expression (Table 1). In our validated cohort, high $L A M B 3$ expression corresponds to more lymph node metastasis

Table I The relationship between LAMB3 expression and clinicopathologic features in TCGA cohort

\begin{tabular}{|c|c|c|c|}
\hline $\begin{array}{l}\text { Clinicopathologic } \\
\text { features }\end{array}$ & $\begin{array}{l}\text { Low expression, } \\
n=\mid 87(\%)\end{array}$ & $\begin{array}{l}\text { High expression, } \\
n=188(\%)\end{array}$ & $P$-value \\
\hline \multicolumn{4}{|l|}{ Age (years) } \\
\hline Mean & $47.7 \pm 14.3$ & $45.3 \pm 16.2$ & \\
\hline$<45$ & $84(44.9)$ & $92(48.9)$ & \\
\hline$\geq 45$ & $103(55.1)$ & $96(51.1)$ & 0.436 \\
\hline \multicolumn{4}{|l|}{ Gender } \\
\hline Male & $50(26.7)$ & $48(25.5)$ & \\
\hline Female & I37 (73.3) & I 40 (74.5) & 0.79 \\
\hline \multicolumn{4}{|l|}{ Tumor size $(\mathrm{cm})$} \\
\hline Mean & $2.83 \pm 1.70$ & $2.99 \pm 1.72$ & \\
\hline$\leq 1$ & $17(9.1)$ & $12(6.4)$ & \\
\hline$>1$ & $170(90.9)$ & $176(93.6)$ & 0.326 \\
\hline \multicolumn{4}{|c|}{ Lymph node metastasis } \\
\hline No & $103(55.1)$ & $61(32.4)$ & \\
\hline Yes & $84(44.9)$ & $127(67.6)$ & $<0.001$ \\
\hline \multicolumn{4}{|l|}{ Histological type } \\
\hline Classical type & $116(62.0)$ & $152(80.9)$ & \\
\hline Others & $71(38.0)$ & $36(19.1)$ & $<0.001$ \\
\hline \multicolumn{4}{|l|}{ Clinical stage } \\
\hline$I+I I$ & 127 (67.9) & $108(57.4)$ & \\
\hline III + IV & $60(32.1)$ & $80(42.6)$ & 0.036 \\
\hline
\end{tabular}

Abbreviation: TCGA, The Cancer Genome Atlas.
Table 2 The relationship between LAMB3 expression and clinicopathologic features in the validated cohort

\begin{tabular}{|c|c|c|c|}
\hline $\begin{array}{l}\text { Clinicopathologic } \\
\text { features }\end{array}$ & $\begin{array}{l}\text { Low expression, } \\
n=28(\%)\end{array}$ & $\begin{array}{l}\text { High expression, } \\
\mathrm{n}=\mathbf{2 8}(\%)\end{array}$ & $P$-value \\
\hline \multicolumn{4}{|l|}{ Age (years) } \\
\hline Mean & $49.5 \pm 10.9$ & $45.8 \pm 14.2$ & \\
\hline$<45$ & $8(28.6)$ & II (39.3) & \\
\hline$\geq 45$ & $20(71.4)$ & $17(60.7)$ & 0.397 \\
\hline \multicolumn{4}{|l|}{ Gender } \\
\hline Male & $3(10.7)$ & $8(28.6)$ & \\
\hline Female & $25(89.3)$ & $20(7 \mathrm{I} .4)$ & 0.093 \\
\hline \multicolumn{4}{|l|}{ Tumor size $(\mathrm{cm})$} \\
\hline Mean & $1.36 \pm 0.94$ & $1.62 \pm 0.60$ & \\
\hline$\leq 1$ & $18(64.3)$ & $4(14.3)$ & \\
\hline$>1$ & $10(35.7)$ & $24(85.7)$ & $<0.001$ \\
\hline \multicolumn{4}{|c|}{ Lymph node metastasis } \\
\hline No & II (39.3) & $5(17.9)$ & \\
\hline Yes & $17(60.7)$ & $23(82.1)$ & 0.018 \\
\hline \multicolumn{4}{|l|}{ Clinical stage } \\
\hline$I+I I$ & II (39.3) & $13(46.4)$ & \\
\hline III + IV & $17(60.7)$ & I5 (53.6) & 0.598 \\
\hline
\end{tabular}

$(P=0.018)$ and tumor size $(P<0.001$; Table 2$)$; meanwhile, lymph node metastasis risk was consistent with the previous finding in TCGA. However, we found that there is no significant association between $L A M B 3$ expression and gender, as well as histological type in both cohorts $(P>0.05)$.

\section{Upregulation of LAMB3 increased lymph node metastasis risk of PTC}

To investigate whether the expression level of $L A M B 3$ is associated with lymph node metastasis, we analyzed the relationship between them by logistic regression. Univariate logistic regression analysis indicated that the significant variables for lymph node metastasis were $L A M B 3$ expression (odds ratio [OR] 2.553, 95\% CI 1.678-3.885, $P<0.001$ ), age (OR 0.646, 95\% CI 0.428-0.976, $P=0.038$ ), gender (OR $0.501,95 \%$ CI $0.308-0.815, P=0.005$ ), tumor size (OR 2.242, 95\% CI 1.028-4.889, $P=0.042$ ), and histological type (OR 0.358, 95\% CI 0.226-0.569, $P<0.001$; Table 3). Multivariate logistic analysis using all parameters

Table 3 Univariate logistic regression analysis for the lymph node metastatic risk

\begin{tabular}{llll}
\hline $\begin{array}{l}\text { Clinicopathologic } \\
\text { features }\end{array}$ & OR & $\mathbf{9 5 \%} \mathbf{C l}$ & P-value \\
\hline LAMB3 expression & 2.553 & $1.678-3.885$ & $<0.001$ \\
Age & 0.646 & $0.428-0.976$ & 0.038 \\
Gender & 0.501 & $0.308-0.815$ & 0.005 \\
Tumor size & 2.242 & $1.028-4.889$ & 0.042 \\
Histological type & 0.358 & $0.226-0.569$ & $<0.001$ \\
\hline
\end{tabular}

Abbreviation: OR, odds ratio. 
Table 4 Multivariate logistic regression analysis for the lymph node metastatic risk

\begin{tabular}{llll}
\hline $\begin{array}{l}\text { Clinicopathologic } \\
\text { features }\end{array}$ & OR & $\mathbf{9 5 \%} \mathbf{C l}$ & P-value \\
\hline LAMB3 expression & 2.28 & $1.465-3.549$ & $<0.001$ \\
Age & 0.671 & $0.433-1.04$ & 0.074 \\
Gender & 0.476 & $0.284-0.796$ & 0.005 \\
Tumor size & 2.043 & $0.911-4.584$ & 0.083 \\
Histological type & 0.444 & $0.274-0.721$ & 0.001 \\
\hline
\end{tabular}

Abbreviation: OR, odds ratio.

also revealed that $L A M B 3$ expression (OR 2.280, 95\% CI $1.465-3.549, P<0.001$ ), gender (OR 0.476, 95\% CI $0.284-0.796, P=0.005$ ), and histological type (OR 0.444, 95\% CI $0.274-0.721, P<0.001$ ) were positively correlated with more lymph node metastasis, whereas age (OR 0.671, 95\% CI 0.433-1.040, $P=0.074$ ) and tumor size (OR 0.911, $95 \%$ CI $0.911-4.584, P=0.083$ ) were negatively associated with lymph node metastasis (Table 4). In summary, upregulated $L A M B 3$ expression could increase the risk of lymph node metastasis in PTC patients.

\section{Downregulation of LAMB3 suppressed cell proliferation and colony formation}

To know the function of $L A M B 3$ in PTC, we downregulated $L A M B 3$ expression in the mRNA level by transfecting
siRNA. After transfection, $L A M B 3$ expression at the mRNA level was significantly downregulated by RT-qPCR (Figure 2A). Subsequently, we performed cell proliferation and colony formation assays in PTC cell lines (TPC1, BCPAP, KTC-1). Downregulation of the LAMB3 group (S1, S2) significantly inhibited cell proliferation compared with the NC group (Figure 2B). The proliferation capacities of the S1 and S2 groups were significantly inhibited and reached statistical significance at the 4 th day compared with the NC group.

To demonstrate the results of cell proliferation assays, we confirmed that downregulation of $L A M B 3$ inhibited cell line proliferation by colony formation assays (Figure 2C and D), which is according to cell proliferation assays.

\section{Downregulation of LAMB3 inhibited migration and invasion}

The situation of primary tumor metastasis is important for prognosis. To confirm whether $L A M B 3$ plays an essential role in the migratory and invasive capacities of PTC cell lines, we performed migration and invasion assays. As shown in Figure 3A and B, downregulation of the $L A M B 3$ group (S1, S2) inhibited the migration abilities of cell lines compared with the NC group. More distinct results are shown in TPC1 and BCPAP than KTC-1. As shown

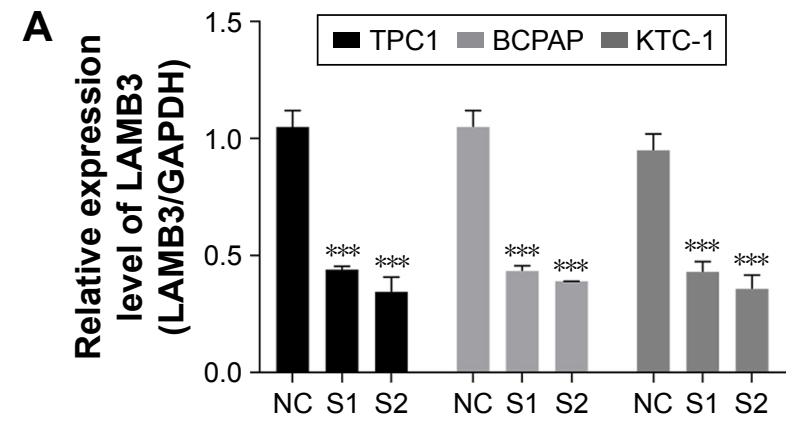

B

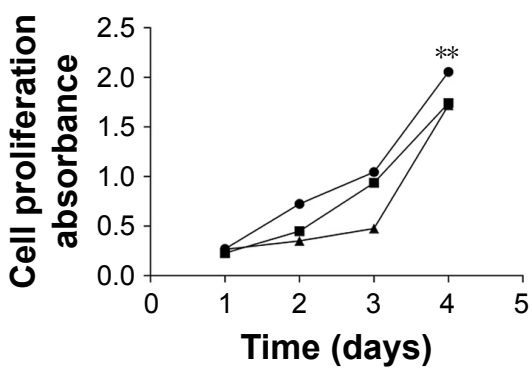

TPC1

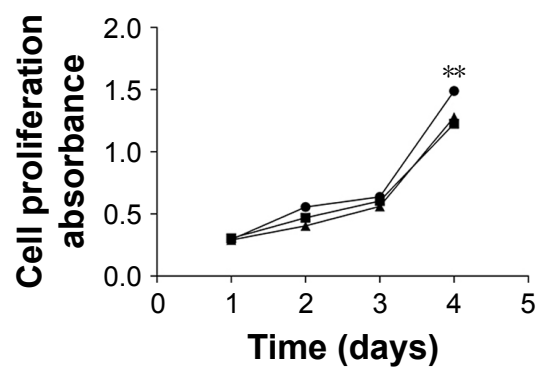

- $\mathrm{NC}-\mathrm{S} 1 \neq \mathrm{S} 2$
KTC-1

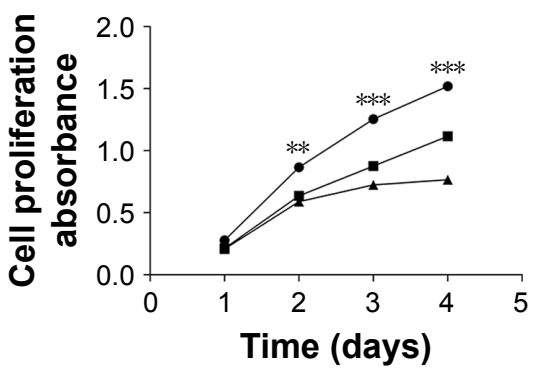

Figure 2 (Continued) 

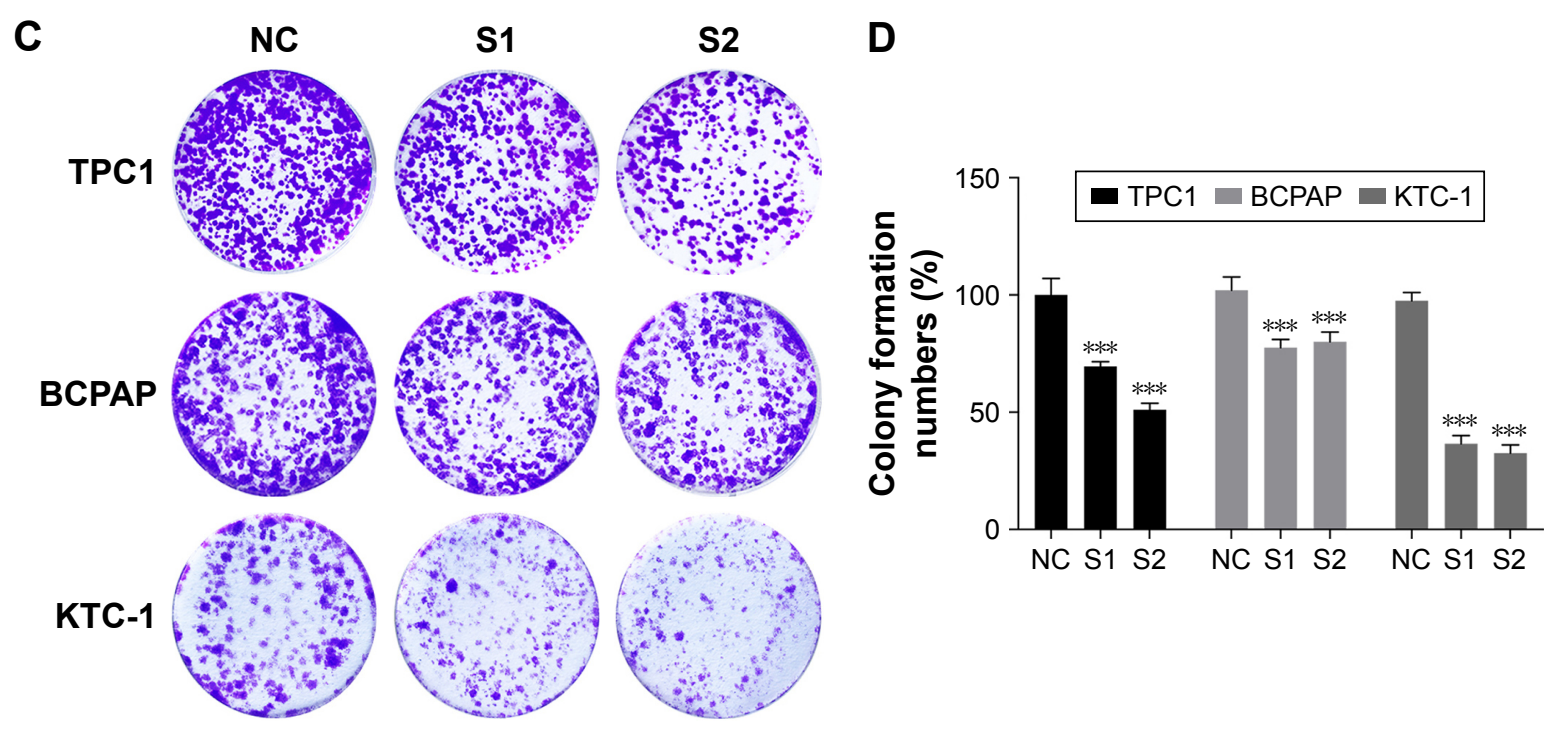

Figure 2 Knockdown of LAMB3 gene inhibited proliferation and colony formation in PTC cell lines.

Notes: (A) LAMB3 relative expression was detected in TPCI, BCPAP, and KTC-I cell lines transfected with siRNA by RT-qPCR (***P $<0.0 \mathrm{I}$ ). (B) Cell proliferation assay. TPCI, BCPAP, and KTC-I cells transfected with siRNA or NC cells were cultured in 96 -well plates for the successive time (I-5 days), and cell proliferation was measured using CCK-8. Cell proliferation was significantly suppressed in TPCI, BCPAP, and KTC-I cells transfected with siRNA compared with NC. (C) Colony formation assay. TPCI, BCPAP, and KTC-I cells transfected with siRNA and NC cells were plated in six-well plates at the proper density. After 8-I4 days of incubation, cells were fixed with $4 \%$ paraformaldehyde and stained with $0.01 \%$ crystal violet. (D) Relative quantification of the colony number. The columns represent the mean colony number from at least three independent experiments, and the little vertical bars on the top of the columns represent SD. $* * P<0.05$, $* * * P<0.0$ I in comparison with NC using Student's $t$-test.

Abbreviations: CCK-8, Cell Counting Kit-8; NC, negative control; PTC, papillary thyroid cancer; RT-qPCR, real-time quantitative polymerase chain reaction; siRNA, small interfering RNA.

in Figure 3C and D, downregulation of the $L A M B 3$ group (S1, S2) inhibited the invasive abilities of cell lines compared with the NC group. The result of invasion assays was consistent with migration. The migration and invasion assays demonstrated that the LAMB3 gene is an important gene associated with PTC-inducing tumor metastasis in PTC.

\section{Discussion}

"PTC" is the most common histological type of thyroid cancer, and "most PTC" patients have a good prognosis with up to $95 \%$ survival after 10 years; however, approximately $20 \%$ of patients relapse after initial treatment. In addition, $5 \%-10 \%$ of patients who relapse may die from the disease because of the failure to respond to conventional therapies. ${ }^{24,25}$ Therefore, knowing the molecular mechanism of PTC is important to predict the occurrence of thyroid cancer and guide its treatment.

LMs are large extracellular glycoproteins that are important components of all basement membrane zones (BMZs) and are involved in several important biological processes, ${ }^{26}$ including wound healing, tissue development, and tumorigenesis. ${ }^{19}$ In normal tissue, the main role of $L M-332$ (also termed $L M-5$ ) is in the maintenance of epithelial-mesenchymal cohesion. ${ }^{27}$ Herlitz's junctional epidermolysis bullosa
(JEB) is one kind of severe and lethal blistering disease, owing to $L M-332$ gene mutations inducing the absence of $L M-332{ }^{28,29}$ In tumor tissues, $L M-332$ stimulated the migration of various cells including carcinoma cells. ${ }^{30-32}$ In gastric carcinoma cells, $L M-332$ could promote migration through mitogen-activated protein kinase (MAPK) pathway activation. ${ }^{33}$ Some researchers demonstrated that $L M-332$ can interact with cell surface receptors, such as EGFR, syndecan 1, and integrins, to promote tumorigenesis in squamous cell carcinoma. ${ }^{19}$

In addition, $L A M B 3$ encodes the $\beta 3$ subunit of $L M-332$. Also, the $L A M B 3$ gene is strongly associated with human cancers. In previous reports, $L A M B 3$ is overexpressed in PTC upon microarray analysis. ${ }^{34}$ Moreover, $L A M B 3$ can be aberrantly upregulated by promoter demethylation and induce gastric cancer..$^{35}$ Furthermore, LAMB3 is related to lymphatic metastasis in lung cancer and affects the migration and invasion capacity in lung cancer cell lines. ${ }^{36}$ In esophageal squamous cell carcinoma, the expression of $L A M B 3$ is significantly associated with the COL7A1 gene, which is useful for predicting the malignant properties of esophageal squamous cell carcinoma. ${ }^{37}$ Besides, in the model of human epidermal carcinogenesis, $L A M B 3$ activates PI3K through interactions with collagen VI. ${ }^{38}$ Some researchers found that osteopontin and $L A M B 3$ can bind to ITGB1, which 


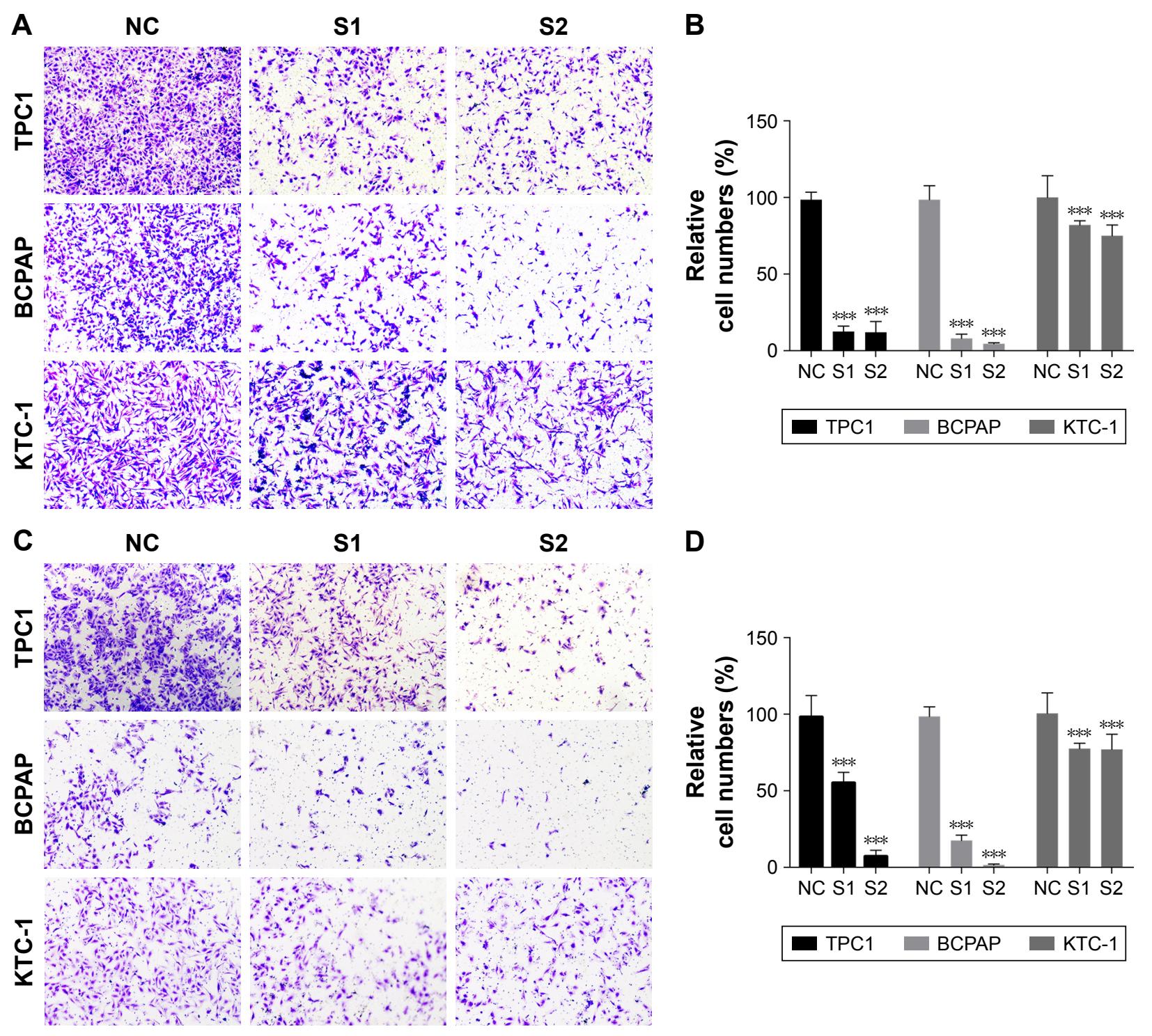

Figure 3 Knockdown of LAMB3 gene inhibited migration and invasion in PTC cell lines.

Notes: Migration assay: (A) migrating cell number was much less in TPCI, BCPAP, and KTC-I cells transfected with siRNA compared with NC; (B) relative quantification of migration cell number. Invasion assay: (C) invading cell number was much less in TPCI, BCPAP, and KTC-I cells transfected with siRNA compared with NC; (D) relative quantification of invasion cell number. Columns represent the mean of cell numbers from at least three independent experiments, and the little vertical bars at the top of the columns represent $\mathrm{SD}$. $* * * P<0.0 \mathrm{I}$ in comparison with NC using Student's $t$-test.

Abbreviations: NC, negative control; PTC, papillary thyroid cancer; siRNA, small interfering RNA.

are receptors for cellular adhesion to the ECM and affect adhesion in the microenvironment to enhance the migration and invasion of highly metastatic cells. ${ }^{36}$ These studies indicated that $L A M B 3$ is an important gene associated with PTC that may affect the tumorigenesis and progression of different tumors. $L A M B 3$ may affect PI3K in thyroid cancer to regulate thyroid cancer cells migration and proliferation which is still unclear, we will investigate this hypothesis in our further study.

Ultrasonography and cytological examination by fineneedle aspiration biopsy (FNAB) are the most common approaches to differentiate malignant and benign thyroid nodules. Even cytological examination, being the most accurate preoperative diagnostic method, cannot differentiate all cases. In addition, $10 \%-26 \%$ of FNABs of thyroid nodules are diagnosed as indeterminate, and $1 \%-11 \%$ are nondiagnostic. ${ }^{39,40}$ With the development of high-throughput technologies, previous studies have created some diagnostic panels from microarray and next-generation sequence data to deal with this urgent clinical problem. Some researchers developed a diagnostic molecular panel consisting of 167 genes, with sensitivity and specificity of $92 \%$ and $52 \%$, respectively. ${ }^{41}$ Using next-generation sequencing, another study established the ThyroSeq V2 assay, which includes more than 50 gene 
mutations and gene fusions, with high sensitivity (90\%) and specificity (93\%).42 The discovery of the gene markers is necessary to differentiate indeterminate thyroid nodules.

In our study, $L A M B 3$ expression at the RNA level was significantly upregulated by RNA-seq of 19 pairs of matched PTC tissues and normal tissues, according to data from TCGA. Moreover, we collected 89 pairs of thyroid nodules and matched adjacent normal tissues (56 pairs of PTC and 33 pairs of benign thyroid nodules) and demonstrated that $\angle A M B 3$ is upregulated in PTC but not in benign thyroid nodules. Subsequently, we demonstrated that upregulated $L A M B 3$ is closely related to lymph node metastasis which is validated by TCGA data set and our tissue specimens. To explore the biological function of $L A M B 3$, we chose three PTC cell lines (TPC1, BCPAP, and KTC-1). By knocking down $L A M B 3$ expression in the RNA level by siRNA, we demonstrated that cell proliferation, migration, and invasion were significantly inhibited.

Our study has limitations. Although we found the potential diagnostic value of $L A M B 3$ in differentiating indeterminate thyroid nodules by postoperative specimens, $L A M B 3$ expression in FNAB and diagnostic value were not evaluated. Besides, the specific mechanisms of $L A M B 3$ in PTC remain to be further investigated.

\section{Conclusion}

By using RNA-seq and bioinformatics analysis, we discovered that $L A M B 3$ is a gene associated with PTC, which is closely related to lymph node metastasis. Subsequently, we demonstrated that the downregulation of $L A M B 3$ can inhibit cell proliferation, migration, and invasion. Our study provided a new potential diagnostic biomarker and therapeutic target for PTC.

\section{Acknowledgment}

This study was funded by the National Natural Science Foundation of China (No 81372380).

\section{Disclosure}

The authors report no conflicts of interest in this work.

\section{References}

1. Pellegriti G, Frasca F, Regalbuto C, Squatrito S, Vigneri R. Worldwide increasing incidence of thyroid cancer: update on epidemiology and risk factors. J Cancer Epidemiol. 2013;2013:965212.

2. Conzo G, Mauriello C, Docimo G, et al. Clinicopathological pattern of lymph node recurrence of papillary thyroid cancer. Implications for surgery. Int J Surg. 2014;12(suppl 1):S194-S197.

3. Morris LG, Sikora AG, Tosteson TD, Davies L. The increasing incidence of thyroid cancer: the influence of access to care. Thyroid. 2013; 23(7):885-891.
4. Enewold L, Zhu K, Ron E, et al. Rising thyroid cancer incidence in the United States by demographic and tumor characteristics, 1980-2005. Cancer Epidemiol Biomarkers Prev. 2009;18(3):784-791.

5. Morris LG, Myssiorek D. Improved detection does not fully explain the rising incidence of well-differentiated thyroid cancer: a populationbased analysis. Am J Surg. 2010;200(4):454-461.

6. Gambardella C, Tartaglia E, Nunziata A, et al. Clinical significance of prophylactic central compartment neck dissection in the treatment of clinically node-negative papillary thyroid cancer patients. World $J$ Surg Oncol. 2016;14(1):247.

7. Hundahl SA, Fleming ID, Fremgen AM, Menck HR. A National Cancer Data Base report on 53,856 cases of thyroid carcinoma treated in the U.S., 1985-1995 [see comments]. Cancer. 1998;83(12):2638-2648.

8. Nikiforov YE. RET/PTC rearrangement in thyroid tumors. Endocr Pathol. 2002;13(1):3-16.

9. Soares P, Lima J, Preto A, et al. Genetic alterations in poorly differentiated and undifferentiated thyroid carcinomas. Curr Genomics. 2011; 12(8):609-617.

10. Elisei R, Viola D, Torregrossa L, et al. The BRAF(V600E) mutation is an independent, poor prognostic factor for the outcome of patients with low-risk intrathyroid papillary thyroid carcinoma: singleinstitution results from a large cohort study. J Clin Endocrinol Metab. 2012;97(12):4390-4398.

11. Tavares C, Melo M, Cameselle-Teijeiro JM, Soares P, Sobrinho-Simoes M. Endocrine tumours: genetic predictors of thyroid cancer outcome. Eur $J$ Endocrinol. 2016;174(4):R117-R126.

12. Millington GW. Mutations of the BRAF gene in human cancer, by Davies, et al. (Nature. 2002;417:949-954). Clin Exp Dermatol. 2013; 38(2):222-223.

13. Crescenzi A, Trimboli P, Modica DC, et al. Preoperative assessment of TERT promoter mutation on thyroid core needle biopsies supports diagnosis of malignancy and addresses surgical strategy. Horm Metab Res. 2016;48(3):157-162.

14. Kimura ET, Nikiforova MN, Zhu Z, Knauf JA, Nikiforov YE, Fagin JA. High prevalence of BRAF mutations in thyroid cancer: genetic evidence for constitutive activation of the RET/PTC-RAS-BRAF signaling pathway in papillary thyroid carcinoma. Cancer Res. 2003;63(7):1454-1457.

15. Sobrinho-Simoes M, Maximo V, Rocha AS, et al. Intragenic mutations in thyroid cancer. Endocrinol Metab Clin North Am. 2008;37(2): 333-362, viii.

16. Horn S, Figl A, Rachakonda PS, et al. TERT promoter mutations in familial and sporadic melanoma. Science. 2013;339(6122):959-961.

17. Melo M, da Rocha AG, Vinagre J, Sobrinho-Simoes M, Soares P. Coexistence of TERT promoter and BRAF mutations in papillary thyroid carcinoma: added value in patient prognosis? J Clin Oncol. 2015;33(6): $667-668$.

18. Morita N, Ikeda Y, Takami H. Clinical significance of $\mathrm{p} 53$ protein expression in papillary thyroid carcinoma. World J Surg. 2008;32(12): 2617-2622.

19. Marinkovich MP. Tumour microenvironment: laminin 332 in squamouscell carcinoma. Nat Rev Cancer. 2007;7(5):370-380.

20. Aumailley M, Bruckner-Tuderman L, Carter WG, et al. A simplified laminin nomenclature. Matrix Biol. 2005;24(5):326-332.

21. Miner JH, Yurchenco PD. Laminin functions in tissue morphogenesis. Annu Rev Cell Dev Biol. 2004;20:255-284.

22. Zhou X, Chen X, Hu L, et al. Polymorphisms involved in the miR-218LAMB3 pathway and susceptibility of cervical cancer, a case-control study in Chinese women. Gynecol Oncol. 2010;117(2):287-290.

23. Reis ST, Timoszczuk LS, Pontes-Junior J, et al. The role of micro RNAs let7c, 100 and 218 expression and their target RAS, C-MYC, BUB1, RB, SMARCA5, LAMB3 and Ki-67 in prostate cancer. Clinics (Sao Paulo). 2013;68(5):652-657.

24. Handkiewicz-Junak D, Czarniecka A, Jarzab B. Molecular prognostic markers in papillary and follicular thyroid cancer: current status and future directions. Mol Cell Endocrinol. 2010;322(1-2):8-28.

25. Davies L, Welch HG. Current thyroid cancer trends in the United States. JAMA Otolaryngol Head Neck Surg. 2014;140(4):317-322. 
26. Sasaki T, Fassler R, Hohenester E. Laminin: the crux of basement membrane assembly. J Cell Biol. 2004;164(7):959-963.

27. Ryan MC, Christiano AM, Engvall E, et al. The functions of laminins: lessons from in vivo studies. Matrix Biol. 1996;15(6):369-381.

28. Meneguzzi G, Marinkovich MP, Aberdam D, Pisani A, Burgeson R, Ortonne JP. Kalinin is abnormally expressed in epithelial basement membranes of Herlitz's junctional epidermolysis bullosa patients. Exp Dermatol. 1992;1(5):221-229.

29. Kivirikko S, McGrath JA, Baudoin C, et al. A homozygous nonsense mutation in the alpha 3 chain gene of laminin 5 (LAMA3) in lethal (Herlitz) junctional epidermolysis bullosa. Hum Mol Genet. 1995;4(5): 959-962.

30. Miyazaki K, Kikkawa Y, Nakamura A, Yasumitsu H, Umeda M. A large cell-adhesive scatter factor secreted by human gastric carcinoma cells. Proc Natl Acad Sci U S A. 1993;90(24):11767-11771.

31. Lenander C, Habermann JK, Ost A, et al. Laminin-5 gamma 2 chain expression correlates with unfavorable prognosis in colon carcinomas. Anal Cell Pathol. 2001;22(4):201-209.

32. Giannelli G, Falk-Marzillier J, Schiraldi O, Stetler-Stevenson WG, Quaranta V. Induction of cell migration by matrix metalloprotease-2 cleavage of laminin-5. Science. 1997;277(5323):225-228.

33. Kariya Y, Miyazaki K. The basement membrane protein laminin-5 acts as a soluble cell motility factor. Exp Cell Res. 2004;297(2): 508-520.

34. Aldred MA, Huang Y, Liyanarachchi S, et al. Papillary and follicular thyroid carcinomas show distinctly different microarray expression profiles and can be distinguished by a minimum of five genes. J Clin Oncol. 2004;22(17):3531-3539.
35. Kwon OH, Park JL, Kim M, et al. Aberrant up-regulation of LAMB3 and LAMC2 by promoter demethylation in gastric cancer. Biochem Biophys Res Commun. 2011;406(4):539-545.

36. Wang XM, Li J, Yan MX, et al. Integrative analyses identify osteopontin, LAMB3 and ITGB1 as critical pro-metastatic genes for lung cancer. PLoS One. 2013;8(2):e55714.

37. Kita Y, Mimori K, Tanaka F, et al. Clinical significance of LAMB3 and COL7A1 mRNA in esophageal squamous cell carcinoma. Eur $J$ Surg Oncol. 2009;35(1):52-58.

38. Waterman EA, Sakai N, Nguyen NT, et al. A laminin-collagen complex drives human epidermal carcinogenesis through phosphoinositol-3kinase activation. Cancer Res. 2007;67(9):4264-4270.

39. Schneider DF, Chen H. New developments in the diagnosis and treatment of thyroid cancer. CA Cancer J Clin. 2013;63(6):374-394.

40. Xing M, Haugen BR, Schlumberger M. Progress in molecular-based management of differentiated thyroid cancer. Lancet. 2013;381(9871): 1058-1069.

41. Alexander EK, Kennedy GC, Baloch ZW, et al. Preoperative diagnosis of benign thyroid nodules with indeterminate cytology. N Engl J Med. 2012;367(8):705-715.

42. Nikiforov YE, Carty SE, Chiosea SI, et al. Highly accurate diagnosis of cancer in thyroid nodules with follicular neoplasm/suspicious for a follicular neoplasm cytology by ThyroSeq v 2 next-generation sequencing assay. Cancer. 2014;120(23):3627-3634.
OncoTargets and Therapy

\section{Publish your work in this journal}

OncoTargets and Therapy is an international, peer-reviewed, open access journal focusing on the pathological basis of all cancers, potential targets for therapy and treatment protocols employed to improve the management of cancer patients. The journal also focuses on the impact of management programs and new therapeutic agents and protocols on

\section{Dovepress}

patient perspectives such as quality of life, adherence and satisfaction The manuscript management system is completely online and includes a very quick and fair peer-review system, which is all easy to use. Visit http://www.dovepress.com/testimonials.php to read real quotes from published authors. 